\title{
Analysis of an Unusual Cluster of Systemic Reactions to Subcutaneous Specific Immunotherapy with Respiratory Allergens in One Allergy Center
}

\author{
Kathrin Scherer ${ }^{a} \quad$ Christian Schindler $^{b} \quad$ Andreas Arnold $^{a} \quad$ Silvy Bach $^{a}$ \\ Andreas J. Bircher ${ }^{\mathrm{a}}$

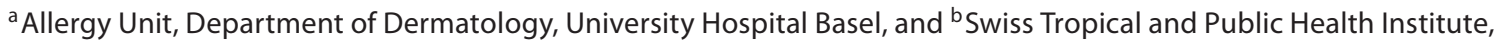 \\ Basel, Switzerland
}

\section{Key Words}

Subcutaneous immune therapy • Respiratory allergen •

Systemic reaction

\begin{abstract}
Systemic reactions to subcutaneous immunotherapy occur despite all necessary precautions and experienced staff and should prompt a search for causative factors. We present an analysis of 11 reactions, 8 of them within a short period. The patients and reactions were evaluated regarding extract errors (composition, concentration), dosing errors, ignored contraindications to specific subcutaneous immunotherapy, introductions versus maintenance phase and accidental intravascular injection. No single or common cause could be identified. Statistical analysis suggests that exceptional clusters of systemic reactions such as these may be just random cumulations without identifiable cause.
\end{abstract}

Copyright $\odot 2011$ S. Karger AG, Base

Specific subcutaneous immunotherapy (SCIT) with respiratory allergens is well established. However, local as well as systemic reactions (SR) and, in rare cases, fatalities $[1,2]$ are possible. Rates of non-fatal SR vary, depending on the allergen type and extracts, the type of immunotherapy (standard vs. cluster vs. rush) and induction versus maintenance phase $[3,4]$. Retrospectively, they range from 0.01 to $0.37 \%[5,6]$, prospectively, around $0.1 \%$ [7] of injections. The rate of SR per patient varies from 1.1 to $7.4[5,8]$, and most are grade I and II reactions.

In our allergy unit, SCIT is routinely performed. Approximately $120-160$ patients per year are regularly started with respiratory allergens by standard and cluster regimens. Experienced nurse staff prepares the extract solutions and allergists perform the injections. Patients are surveyed for at least $30 \mathrm{~min}$. If SR are encountered they are treated immediately.

In 2004, we treated 158 patients and we observed a total of 11 SR in 10 patients $(6.32 \%$ of patients, 8 females, mean age 32 years, range $18-71 ; 8$ patients had preexisting, currently well-controlled asthma). A cluster of 8 SR occurred in the last quarter; 5 patients suffered from 6 reactions within a period of 6 weeks. Seven of 11 SR occurred to Novo-Helisen ${ }^{\circledR}$ (Allergopharma, Reinbek, Germany), 3 to Alutard SQ ${ }^{\circledR}$ (ALK Denmark) and 1 to an Allergovit ${ }^{\circledR}$ (Allergopharma) preparation. Eight of the 10 patients were treated with 2 different extracts, 2 patients with 1 extract each. One patient with 2 extracts had 2 SR. All SR occurred within $30 \mathrm{~min}$ after the injection. The

\section{KARGER}

Fax +41613061234 E-Mail karger@karger.ch www.karger.com
(C) 2011 S. Karger AG, Base

$1018-2438 / 12 / 1573-0318 \$ 38.00 / 0$

Accessible online at:

www.karger.com/iaa
Correspondence to: Prof. Andreas J. Bircher

Allergy Unit, Department of Dermatology, University Hospital Basel

Petersgraben 4

$\mathrm{CH}-4031$ Basel (Switzerland)

Tel. +41 61265 4359, E-Mail birchera@ uhbs.ch 
Table 1. Number of sessions, allergen extracts and doses per quarter in 2004

\begin{tabular}{llllll}
\hline & 1. quarter & 2. quarter & 3. quarter & 4. quarter & Total \\
\hline Sessions, n & 287 & 374 & 301 & 596 & $1,444(100 \%)$ \\
Allergovit doses & 115 & 50 & 0 & 135 & 300 \\
Alutard SQ doses & 162 & 182 & 167 & 247 & 559 \\
Novo-Helisen doses & 406 & 303 & 322 & 599 & $1,630(85.5 \%)$ \\
\hline Grades of AR & & & & Patients with AR \\
\hline Allergovit & 0 & 0 & 0 & I & 1 \\
Alutard SQ & 0 & 0 & 0 & II & 1 \\
$\quad$ Induction & & III & III & 0 & 2 \\
Maintenance & 0 & 0 & 0 & II, III, IV & 3 \\
$\quad$ Induction & & II & 0 & I, III, III & 4 \\
$\quad$ Maintenance & & 2 & 1 & 8 & 11 \\
\hline Total adverse reactions & & & & & \\
\hline
\end{tabular}

$\mathrm{AR}=$ Adverse reactions; $\mathrm{I}=\mathrm{AR}$ grade $\mathrm{I}$ II = AR grade II; III = AR grade III; IV = AR grade IV .

reactions were graded according to H.L. Mueller: 2 grade I, 3 grade II, 5 grade III and 1 grade IV. One patient suffered from a grade II and a grade III reaction within 1 month (table 1). Treatment included inhaled and intramuscular epinephrine, intravenous clemastine and methylprednisolone, with a beneficial response in all. All cases were reported to the pharmacovigilance and documented as critical incidence reports.

We retrospectively identified all patients who had received specific immunotherapy with respiratory allergens, the products used and the number of injections given. In 2004, 158 patients with rhinoconjunctivitis and/or asthma received a total of 1,630 allergen doses of NovoHelisen (Allergopharma) (table 1), 559 doses of Alutard SQ (ALK Denmark) and 300 doses of Allergovit (Allergopharma). A total of 20 extracts was administered to 10 patients before the $11 \mathrm{SR}$. The calculated incidence of SR was $6.3 \%$ in the patients, and the respective proportions in the preparations were: in 6 patients, Novo-Helisen $14 / 1,630$ doses (0.0066\%), in 3 patients, Alutard SQ 4/559 doses $(0.007 \%)$, and in 1 patient Allergovit $1 / 300$ doses (0.0033\%).

The patients and reactions were evaluated with regard to the following risk factors: (1) extract error (composition, concentration), (2) dosing errors, (3) ignored contraindications (symptoms to allergen exposure, uncontrolled asthma, intercurrent infections, interfering drugs), (4) introduction versus maintenance phase, and (5) accidental intravascular injection.

A Coincidental Cluster of SR to SCIT
No errors with regard to extract selection or dosing could be identified. Injections were given subcutaneously on the lateral upper arm after careful aspiration. The 6 patients who had received Novo-Helisen preparations were further analyzed. The administered preparations were sent back to the company and analyzed with regard to allergen content. All solutions contained the respective allergens in correct concentrations. All 10 patients continued SCIT up to a complete 3-year course without further SR.

A logistic regression analysis was performed to assess the statistical significance of the incidence rates between the last 4 weeks of 2004 as compared to the rest of the year. Five of the 11 cases occurred during these last 4 weeks of 2004. The analysis was adjusted for potential heterogeneity of susceptibility between subjects and provided a $p$ value of 0.0002 . To test the hypothesis that nonrandom clustering occurs in at least 1 of the 52 possible 4-week periods against the null-hypothesis of side effects occurring independently of each other and without any association with calendar time, this $\mathrm{p}$ value would have to be multiplied by 52 (Bonferroni adjustment). Therefore, an adjusted $\mathrm{p}$ value of 0.01 might be appropriate. In addition, we conducted a simulation analysis in which we randomly distributed the 11 events across all sessions of the year. As a measure of heterogeneity, we considered the variance of successive distances between events. These distances were defined as the number of sessions between consecutive events augmented by 1 . Closing the 'string' of

Int Arch Allergy Immunol 2012;157:318-320 
sessions to a 'necklace' enabled the determination of an 11 th distance. In less than 10 out of 10,000 randomly generated distributions, the respective variance was higher than the one observed in our data, suggesting that there was even less visible clustering. In summary, the higher incidence of severe allergic reactions observed in the 4 th quarter of 2004, and more specifically during the last month of 2004, can be explained as a rare clustering of random events.

In table 1, the overview of the 4 quarters of 2004 with the number of subcutaneous injections of allergen extracts, the number of sessions and the number of the 3 different preparations used is given.

Novo-Helisen was the most frequently administered extract (85.5\%) with a rate of 7 adverse SR among 1,630 administered doses (0.0066\%). There was no statistical significance with regard to other preparations and no difference between induction and maintenance doses. We further analyzed possible causes of SR to this extract. Neither composition nor dilution or dosing errors nor ignored contraindications could be identified. Since most reactions occurred outside of the pollen season, this factor is most likely not relevant [9]. Having excluded - as far as possible - all known causes for SR $[10,11]$ and hav- ing assured a proper subcutaneous injection technique, we suppose that the question of where the allergen is deposited in the subcutaneous tissue in relation to blood vessels in particular might be of relevance with regard to the speed of systemic distribution of the allergen. However, there are no hard data on this issue. For the cluster of SR in 2004, we suppose that most likely, paravascular injections were responsible for the SR, which began most often within a few minutes after the injection.

According to our estimates, the risk of observing a comparable clustering in a given year just by chance is $<1 \%$ in our setting. In the following year $2005,4.6 \%$ of patients (as compared to $6.32 \%$ in 2004) suffered SR during SCIT. Exact rates per injection were not calculated.

In conclusion, we have seen comparable rates of SR in SCIT as reported in the literature [5]. Particular risk factors such as uncontrolled asthma, cluster treatment or allergen extracts from a particular manufacturer [12-14] were not present. Mix-up of extracts or wrong doses could not be identified. Also, other patient- or physician-related mistakes were not present. Severe allergic reactions occur rarely upon SCIT, and even in a well-controlled, experienced setting, their onset is possible at any time. However, the cluster we observed is a very rare event.

\section{References}

1 Lockey RF, Benedict LM, Turkeltaub PC, Bukantz SC: Fatalities from immunotherapy (IT) and skin testing (ST). J Allergy Clin Immunol 1987;79:660-677.

-2 Bernstein DI, Wanner M, Borish L, Liss GM: Twelve-year survey of fatal reactions to allergen injections and skin testing: 19902001. J Allergy Clin Immunol 2004;113: 1129-1136.

- 3 Winther L, Arnved J, Malling HJ, Nolte H, Mosbech H: Side-effects of allergen-specific immunotherapy: a prospective multi-centre study. Clin Exp Allergy 2006;36:254-260.

-4 Harvey SM, Laurie S, Hilton K, Khan DA: Safety of rush immunotherapy to multiple aeroallergens in an adult population. Ann Allergy Asthma Immunol 2004;92:414-419.

5 Cox L, Larenas-Linnemann D, Lockey RF, Passalacqua G: Speaking the same language: The World Allergy Organization Subcutaneous Immunotherapy Systemic Reaction Grading System. J Allergy Clin Immunol 2010;125:569-574.
6 Ragusa VF, Massolo A: Non-fatal systemic reactions to subcutaneous immunotherapy: a 20-year experience comparison of two 10 year periods. Eur Ann Allergy Clin Immunol 2004;36:52-55.

7 Bernstein DI, Epstein T, Murphy-Berendts K, Liss GM: Surveillance of systemic reactions to subcutaneous immunotherapy injections: year 1 outcomes of the ACAAI and AAAAI collaborative study. Ann Allergy Asthma Immunol 2010;104:530-535.

8 Adamic K, Zidarn M, Bajrovic N, Erzen R, Kopac P, Music E: The local and systemic side-effects of venom and inhaled-allergen subcutaneous immunotherapy. Wien Klin Wochenschr 2009;121:357-360.

-9 Toubi E, Kessel A, Blant A, Golan TD: Follow-up after systemic adverse reactions of immunotherapy. Allergy 1999;54:617-620.
10 Rezvani M, Bernstein DI: Anaphylactic reactions during immunotherapy. Immunol Allergy Clin N Am 2007;27:295-307.

-11 Aaronson DW, Gandhi TK: Incorrect allergy injections: allergists' experiences and recommendations for prevention. J Allergy Clin Immunol 2004;113:1117-1121.

12 Justicia JL, Barasona MJ, Serrano P, Moreno C, Guerra F: Predicting patients at high-risk of systemic reactions to cluster allergen immunotherapy: a pilot prospective observational study. J Invest Allergol Clin Immunol 2006;17:386-392.

13 Gastaminza G, Algorta J, Audicana M, Etxenagusia M, Fernández E, Muñoz D: Systemic reactions to immunotherapy: influence of composition and manufacturer. Clin Exp Allergy 2003;33:470-474.

14 Iglesias-Cadarso A, Hernández-Weigand P, Reaño M, Herrera I, Sánchez-Morillas L, Pérez-Pimiento A: A prospective safety study of allergen immunotherapy in daily clinical practice. Allergol Immunopathol (Madr) 2004;32:278-283. 